\title{
Tip60 Tumor Suppressor Requires Its NLS Motif to Interact with Importin $\alpha$
}

\author{
Eun Jeoung Lee1, Sung Hwa Shin'1, Sang Sun Kang2* \\ ${ }^{1}$ CdmoGen, 530, Jikji-daero, Heungdeok-gu, Cheongju, Chungbuk, Republic of Korea \\ ${ }^{2}$ Department of Biology Education, Chungbuk National University, Chungdae-ro, Seowon-gu, Cheongju, Chungbuk, \\ Republic of Korea \\ Email: ‘jin95324@cbu.ac.kr
}

How to cite this paper: Lee, E.J., Shin, S.H. and Kang, S.S. (2019) Tip60 Tumor Suppressor Requires Its NLS Motif to Interact with Importin $\alpha$. CellBio, 8, 1-16. https://doi.org/10.4236/cellbio.2019.81001

Received: March 6, 2019

Accepted: March 27, 2019

Published: March 30, 2019

Copyright (C) 2019 by author(s) and Scientific Research Publishing Inc. This work is licensed under the Creative Commons Attribution International License (CC BY 4.0).

http://creativecommons.org/licenses/by/4.0/

(c) (i) Open Access

\begin{abstract}
Tip60 is a specific member of MYST (Moz-Ybf2/Sas3-Sas2-Tip60) family of nuclear histone acetyltransferases (HAT). It is essential for cellular survival, differentiation, and metabolism. A putative canonical NLS motif between the chromo domain and the zinc finger of Tip60 was identified. Here we show evidence that Tip60 is associated with importin $\alpha$ as its substrate and transported from cytoplasm to the nucleus. Pull down assay revealed that Tip60 was physically associated with importin $\alpha$ both in vivo and in vitro. Confocal microscopic observation showed that Tip60 and importin $\alpha$ were co-localized with each other. The localization of Tip60 to the nuclear and its interaction with importin $\alpha$ was disrupted when its putative NLS motif for binding to importin $\alpha$ was mutated $\left({ }^{219} \mathrm{RKRK}^{222} \rightarrow{ }^{219} \mathrm{AAAA}^{222}\right)$. However, attachment of this putative NLS motif to a cytoplasmic protein (YAP 1-210 fragment) promoted its nuclear localization. Based on transient transfection, Tip60 NLS motif mutant showed a substantial reduction in self-acetylation, HAT activity, and apoptotic ability whereas wild type Tip60 did not show such reduction. Taken together, our results demonstrate that importin $\alpha$ transports Tip60 from the cytoplasm to the nucleus through binding to the putative NLS motif of Tip60 for its tumor suppressing function.
\end{abstract}

\section{Keywords}

Tip60, Importin $\alpha$, Nuclear Localization Sequence, Protein-Protein Interaction, HAT Activity, Cell Survival

\section{Introduction}

Tip60 (Isoform1: NP874369, 546 aa in length), a protein associated with human immunodeficiency virus type 1-encoded transactivation protein (Tat), has been 
characterized as a specific member of MYST (Moz-Ybf2/Sas3-Sas2-Tip60) family of nuclear histone acetyltransferases (HATs) [1] [2] [3]. To elucidate its biological roles and identify proteins interacted with ectopically expressed Tip60, the presence of associated ATPase and DNA helicase activities has been demonstrated [4] [5]. Functional experiments have revealed that Tip60 and its associated proteins play essential roles in DNA repair and apoptosis [6] [7]. Moreover, Tip60 appears to be related to a variety of cellular functions depending on acetylation of its substrate proteins [4] [8] [9] [10]. Protein acetylation is a modification by acetyltransferases that catalyze the transfer of acetyl groups from acetyl coenzyme A to either $\mathcal{\varepsilon}$-amino group of internal lysine residues or $\mathcal{\varepsilon}$-amino group of N-terminal amino acids [11] [12].

While mechanisms involved in regulation of Tip60 are not fully defined yet, the precise control of its function appears to be achieved through a combination of acetylation, localization, and interaction with Tip60 binding proteins [4] [13]. Even though Tip60 is predominantly located in the nuclei, it is also present in the cytosol and mitochondria [8] [14] [15]. Therefore, the mechanism how subcellular localization of Tip60 is controlled remains unclear. Analysis of its amino acid sequence does not reveal the presence of any recognizable import or export sequences. Others have demonstrated that Tip60 binding proteins also regulate the subcellular localization of Tip60 by inhibiting its nuclear export [1] [8] [11] [12] [13]. Thus, subcellular localization of Tip60 might be indirectly regulated through its association with binding proteins.

Nucleocytoplasmic protein transport is mainly mediated by importin proteins (importins/exportins) [16] [17]. More than 19 human importins and 14 yeast importins have been characterized recently [18] [19]. Among them, importin $\alpha$ can recognize and bind substrates via nuclear canonical localization signals (cNLSs) for transport through the nuclear pore complex [6]-[21]. Once inside the nucleus, imported importin $\alpha$ can bind to small GTPases, such as RanGTPase and release their substrates [22] [23] [24]. Different sets of substrates with distinct NLSs are recognized by several importins [16] [17] [18] [19]. A well-characterized canonical NLS is the short and basic cNLS associated with Kap $\alpha$ /importin $\alpha$ heterodimer (yeast Kap95p) [23] [24]. Monopartite cNLSs consist of a single cluster of basic residues with a consensus sequence of $\mathrm{K}(\mathrm{K} / \mathrm{R}) X(\mathrm{~K} / \mathrm{R})$, while bipartite NLSs have two clusters of basic residues separated by 10-12 amino acids [20] [25] [26]. Thus, the cNLS is a relatively small but well defined NLS that has concentrated binding energy.

Through visual inspection of Tip60 amino acid sequence, it was noticed that Tip60 contains a putative importin $\alpha$ binding motif $\left({ }^{210} \mathrm{RRAVAAQPGRKRK}{ }^{222}\right)$ [20] [23] [24] [25] [26]. Thus, the objective of this study was to determine whether Tip60 could interact with importin $\alpha$, and investigate whether such interaction might be related to the tumor suppressor function of Tip60. Consequently, our study determined at the first time that Tip60 NLS promotes its nuclear localization through binding with importin $\alpha$ and enhances its tumor suppressor function. Our observations might provide the clue on the molecular 
mechanism underlying Tip60 tumor suppressor function which is regulated by its unclear localization.

\section{Materials and Methods}

\subsection{Antibodies}

Monoclonal and polyclonal antibodies against actin, EGFP, importin $\alpha$, Tip60, and acetylation protein were purchased from Santa Cruz Biotech Inc. (Santa Cruz, CA, USA) or Cell Signaling (Boston, MA, USA).

\subsection{Cell Culture and Transfections}

HEK293 cells were cultured in DMEM medium supplemented with 10\% (v/v) heat-inactivated fetal bovine serum (FBS) and $1000 \mathrm{U}$ penicillin-streptomycin (GIBCO BRL). Transfection was conducted with Lipofectamine LTX and Plus Reagent (Invitrogen) in accordance with the manufacturer's instructions.

\subsection{Plasmid Constructs}

Wild type human Tip60 in EGFP-mammalian expression vector or GST-tagged E. coli expression vector was purchased from GeneCopoeia Co. (CA, USA). In order to generate importin $\alpha$-binding motif mutant, Tip60 NLS mutant $\left({ }^{219} \mathrm{RKRK}^{222}\right.$ $\rightarrow{ }^{219} \mathrm{AAAA}^{222}$ ) construct, mutagenic primers (UP: 5'-TGT AAC ATA GTC GCA TTG GCT TAT TTC TTC TAC-3', DOWN: 5'-GGA GTA GAA GAA ATA AGC CAA TGC GAC TAT GTT ACA-3' and up: 5'-ATA GTC CGA TTG CGT GCT TTC TTC GCC TCC AGT GGT-3', DOWN: 5'-CTT CTC ACC ACT GGA GGC GAA GAA AGC ACG CAA TCG-3'), and QuikChange Multi Mutagenesis Kit (Stratagene, West Cedar, TX, USA) were utilized according to the manufacturer's instructions GST-tagged recombinant proteins for Tip60 and NLS mutants were purified from Escherichia coli BL21 (DE3) after performing PCR and cloning. All constructs were confirmed by DNA sequencing.

Human YAP cDNA was obtained from the Korea Human Gene Bank (Gene ID: KIAA0623) and cloned into pEGFP1 vector (Clontech, Palo Alto, CA, USA; EGFP was added to the N-terminal of YAP) with the following primers: forward, 5'-CTCAAGCTTCGAATTCATGGAGGTGGTGGGTGAC-3' and reverse 5'-CATAGCACCGCAACCGTGTGAGAATTCTGCAGTCGAC-3'. For deletion mutant 1-210 aa, the following primers were used: forward, 5'-GCC ATG CTG TCC CAG TAG AAC GTC ACA GCC-3' and reverse, 5'-GGG GGC TGT GAC GTT CTA CTG GGA CAG CAT-3'. For fusion of Tip60 NLS motif $\left({ }^{210} \mathrm{RRAVAAQPGRKRK}{ }^{222}\right)$ to the cytoplasmic protein (C-terminal tail of YAP 1-210 fragment), oligonucleotide 36 mer (5'-CGT AGG GCA GTG GCA GCC CAG CCA GCA CGG AAC CGA AAA-3'; Bioneer, Co, Daejeon, Korea) corresponding to the motif's amino acids was inserted by DNA ligation. The construct was confirmed by DNA sequencing.

\subsection{Expression and Purification of Recombinant Proteins}

GST tagged protein Tip60 WT or Tip0 NLS mutant was expressed in E. coli BL21 
and purified with GST-agarose beads (Amersham Biosciences Co.) according to the manufacturer's instruction. Purified proteins were used for pull down assay with importin $\alpha$.

\subsection{Immunoprecipitation}

Cells were routinely analyzed at 48 hours post transfection. Cells were rinsed with ice-cold phosphate-buffered saline and resuspended in $1 \mathrm{~mL}$ of extraction buffer [10 mM Tris-HCl pH7.4, 1mM EDTA, 5 mM DTT, $100 \mathrm{mM} \mathrm{NaCl}, 1.0 \%$ Triton X-100, $60 \mathrm{mM}$ n-octyl glucoside, $1 \mathrm{mM}$ vanadate, $100 \mu \mathrm{M}$ molybdate, 20 $\mathrm{mM}$ sodium fluoride, and protease inhibitor cocktail (1 tablet per $10 \mathrm{ml}$ extraction buffer)]. Pre-cleaned lysate was incubated with appropriate antibody at $4^{\circ} \mathrm{C}$ for 1 hour. The resulting immune complex was collected on Protein A-Sepharose beads (Pharmacia). Immune complexes were then captured by centrifugation, washed extensively with lysis buffer, and solubilized with $2 \times$ sample buffer prior $10 \%$ SDS-PAGE.

\subsection{Tip60 Pull Down Assay}

Whole cell lysate of HEK293 cells was pre-cleaned with glutathione agarose beads and incubated with $1 \mu \mathrm{g}$ of each glutathione agarose tagged recombinant GST-Tip60 (WT) or NLS mutant at $4{ }^{\circ} \mathrm{C}$ on an end-over-end rotating shaker for 2 hours in order to allow for the association of Tip60 protein with importin $\alpha$. Associated protein complexes were collected using slurry of glutathione agarose beads and washed extensively. After resuspension in $2 \times$ Laemmli sample buffer, samples were subjected to $10 \%$ SDS-PAGE and western blot analysis using importin $\alpha$ antibody [27].

\subsection{Immunoblotting}

Pull down or immunoprecipitated Tip60 was subjected to 10\% SDS-PAGE, transferred to nitrocellulose membranes, incubated in blocking buffer (5\% non-fat dried milk PBS and $0.05 \%$ Tween-20), probed with specific primary antibodies, and incubated with horseradish peroxidase-conjugated secondary antibody. Immune complexes were detected with chemiluminescence western blotting detection system (Pierce, Rockford, IL, USA).

\subsection{Confocal Microscopy}

HEK293 cells were cultured overnight at $60 \%$ confluence on glass slides coated with human fibronectin (SPL, Korea). The next day, cells were transfected with EGFP Tip60 WT or its NLS mutant construct and incubated for an additional 48 hours. Cells were washed several times with ice-cold PBS and fixed with $2 \%$ paraformaldehyde for 10 minutes. Fixed cells were permeabilized with $0.1 \%$ Triton $\mathrm{X}-100$ for 10 minutes and blocked with PBS containing 5\% BSA (Aurion, The Netherlands) and $0.1 \%$ Tween for 2 hours. After incubation with a polyclonal (rabbit) or monoclonal (mouse) antibody against importin $\alpha$ or Tip60 (1:300 in 
$5 \%$ BSA-PBS; Bio-Protocol, Palo Alto, CA, USA) overnight at $4^{\circ} \mathrm{C}$, slides were washed three times with $0.01 \%$ PBS-Tween 20. Alexa Fluor 568 or 488-conjugated donkey anti-rabbit or anti-mouse secondary antibody (Molecular Probes, Inc., Eugene, OR, USA) was used. Confocal microscopy analysis was conducted using a LSM710 (Zeiss, Germany) at the Center for Research Instruments and Experimental Facilities of Chungbuk National University. Pearson's correlation coefficient (PCC) of co-localization between importin $\alpha$ and Tip60 was measured with LSM710 (Zeiss, Germany) [28].

\subsection{FACS Analysis}

EGFP-Tip60 (WT), NLS mutant, or EGFP vector was transfected and the rate of apoptosis was measured using Annexin V-PE apoptosis detection kit I (BD Biosciences, CA, USA) according to the manufacturer's instructions. Cells were vortexed gently and incubated at $25^{\circ} \mathrm{C}$ in the dark for $15 \mathrm{~min}$. FACS analysis was performed using FACS Calibur (BD Science) in the Core Facility of Chungbuk National University within 1 hour after adding $400 \mu \mathrm{l}$ of binding buffer to each tube [8] [9].

\subsection{ELISA for Histone Acetyltransferase (HAT) of Tip60}

Cell lysates were immunoprecipitated as described above except that high salt wash step was omitted. Immuprecipitates were washed twice in HAT assay buffer (50 mM Tris, $\mathrm{pH} 8 / 10 \%$ glycerol/0.1mM EDTA/1mM DTT) and incubated in $60 \mu \mathrm{l}$ of HAT assay buffer containing acetyl-CoA $(100 \mu \mathrm{M})$ and biotinylated histone $\mathrm{H} 4$ peptide $(0.5 \mu \mathrm{g})$ at $30^{\circ} \mathrm{C}$ for $30 \mathrm{~min}$. An aliquot of the reaction was immobilized onto streptavidin plates. Lysine acetylation was detected via HAT ELISA according to the manufacturer's instructions (Biovision Milpitas, CA). In some assays, HAT activity was analyzed with Tip60 immobilized onto NiTA-agarose beads [8] [9].

\subsection{Statistics}

Data are shown as means \pm standard error of the mean (s.e.m.). Statistical significance was performed via Welch's t-test or Student's t-test. Statistically significance was considered at $P<0.05$ [28].

\section{Results}

\subsection{Importin $\alpha$ Interacts with Tip60 through the Putative Nuclear Localization Sequences (NLS) of Tip60}

Putative importin $\alpha$ binding motifs are present in most proteins associated with importin $\alpha$ [23] [24] [25] [26]. Diverse NLS sequences consistently contain a weak consensus motif composed of a loose N-terminal hydrophobic or basic motif (http://www.moseslab.csb.utoronto.ca/NLStradamus/). Based on this information, Tip60 (Isoform1:NP874369) was also found to contain a putative importin $\alpha$-binding motif in between its chromodomain and zinc finger (Figure 1(A)) as 
(A)

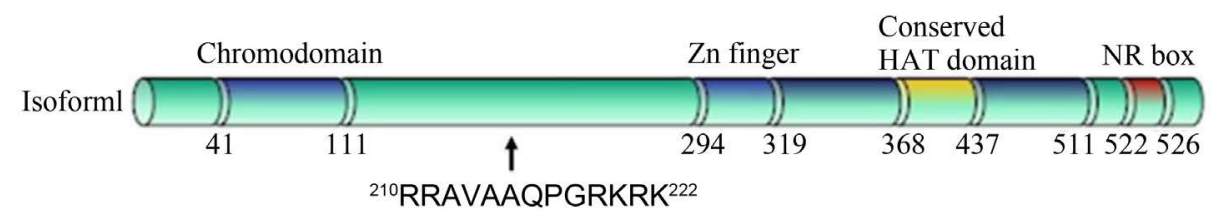

(B)
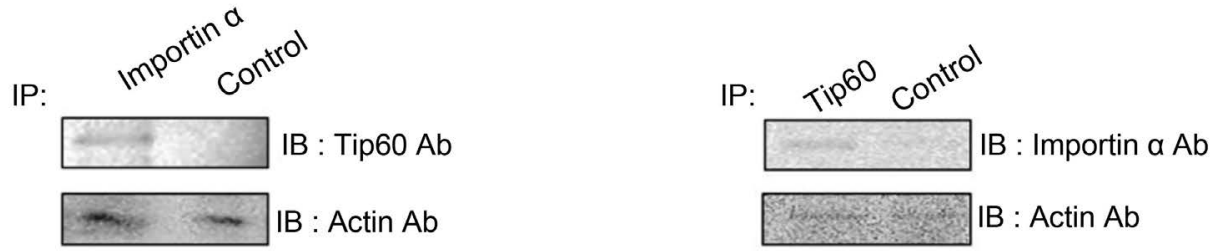

(C)

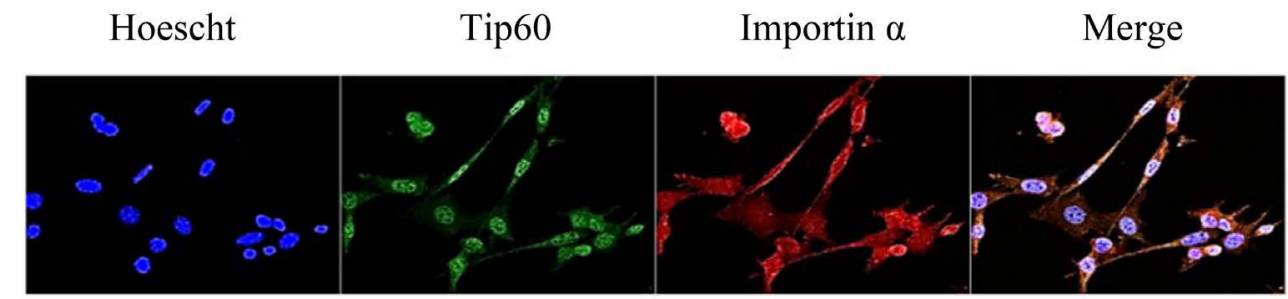

PCC between importina and Ti60 : $0.81+/-0.12(n=10)$

Figure 1. The putative NLS inTip60 and interaction between exogenous Tip60 and importin $\alpha$. (A) Tip60 (Isoform1:NP874369; 546aa) contains the putative-conserved importin $\alpha$ binding motif $\left({ }^{210}\right.$ RRAVAAQPGRKRK $\left.{ }^{222}\right)$ within its binding domain between chromodomain and the zinc finger (yellow). Two point mutants were prepared in order to define the binding region. Tip60 mutant $\left({ }^{219} \mathrm{RKRK}^{222} \rightarrow{ }^{219} \mathrm{AAAA}^{222}\right)$ in the importin $\alpha$ putative binding motifs, were also prepared, and the mutated sequences were indicated as RKRK $\rightarrow$ AAAA $\left({ }^{210}\right.$ RRAVAAQPGRKRK ${ }^{222}$ changes to ${ }^{210}$ RRAVAAQPGAAAA $\left.{ }^{222}\right)$. For these mutants control, we used the unrelated NLS mutant. (B) Following immunoprecipitation (IP) using an anti-importin $\alpha$ antibody, an immunoblot (IB) was performed using an antibody against Tip60 (left). The immunoprecipitated Tip60 complexes were applied to the immunoblot, using an anti-importin $\alpha$ antibody (right). For the negative control, normal mouse serum was used for immunoprecipitation. (C) Confocal fluorescence micrographs showing the endogenous Tip60 and importin $\alpha$ in HEK293 cells. Importin $\alpha$ was visualized by immunofluorescence in fixed and permeablized cells using polyclonal antibodies to human importin $\alpha$ or Tip60 and Alexa Fluor 568 conjugated donkey anti-rabbit IgG or Alexa Fluor 488 conjugated mouse anti-rabbit IgG. The yellow pattern resulting from the merging of red and green colors indicates the co-localization of both proteins at a specific region of the nuclear membrane and nuclear. PCC value between importin $\alpha$ or Tip60 indicates under the confocal pictures.

an NLS [1]. The presence of such putative NLS suggests that Tip60 might be imported by importin $\alpha$.

Since Tip60 appeared to contain a putative importin $\alpha$ binding motif, whether endogenous importin $\alpha$ could form protein complex with Tip60 in HEK293 cells was examined. As shown in Figure 1(B), Tip60 immunoprecipitate was found to contain importin $\alpha$ (right panel). Antibodies directed against importin $\alpha$ were also able to successfully capture Tip60 from the same lysate (Figure 1(B), left panel), corroborating the hypothesis that the two proteins were indeed physically associated. Furthermore, confocal microscopy was performed to determine whether Tip60 was co-localized with importin $\alpha$ in cells. Results are shown in Figure 1(C). Endogenous Tip60 (green) and importin $\alpha$ (red) were indeed 
co-localized in the nucleus (white) and cytoplasm (yellow). Pearson's correlation coefficient (PCC) between importin $\alpha$ and Ti60 was measured to be $0.81+/-$ $0.12(\mathrm{n}=10)$, meaning that $82 \%$ of these two proteins co-existed in the cell. The association between Tip60 and importin $\alpha$ was also observed in COS- 1 and NIH3T3 cells as in HEK 293 cell (data not shown). These results strongly suggest that endogenous Tip60 can interact with importin $\alpha$ in cells.

\subsection{The Interaction between Exogenous Tip60 and Importin $\alpha$ Is Required for Its Nuclear Localization in HEK293 Cells}

To determine whether the putative NLS in Tip60 could interact with importin $\alpha$, Tip60 NLS point mutant $\left({ }^{219} \mathrm{RKRK}^{222} \rightarrow{ }^{219} \mathrm{AAAA}^{222}\right)$ was constructed. After transfection of EGFP vector, EGFP Tip60 WT, or EGFP Tip60 NLS mutant in HEK293 cells, each EGFP- fusion protein was purified with EGFP antibody. To detect protein-protein interaction between Tip60 and importin $\alpha$ (or its acetylation), western blot was conducted with anti-acetylation, anti-importin $\alpha$, or anti-Tip60 antibody. To monitor expression levels of Tip60 and its NLS mutant, western blot analysis was performed using anti-Tip60 antibody. To monitor cell lysate amount, immunoblotting was performed using anti-action antibody (Figure 2(A), bottom lane). As expected, Tip60 WT containing the putative importin $\alpha$ binding motif pulled down importin $\alpha$ from HEK293 cell lysates in high quantities, whereas Tip60 NLS mutant without the putative NLS sequence failed to pull-down appreciable amounts of importin $\alpha$ (Figure 2(A)). Interestingly, EGFP-Tip60 WT was profoundly acetylated (Figure 2(A) top lane). However, Tip60 NLS mutant mainly detected in the cytoplasm was not acetylated (Figure 2(D)), suggesting that Tip60 nuclear localization also associated with its acetylation.

(A)

IP : EGFP Ab

(B)

Purified with GST bead

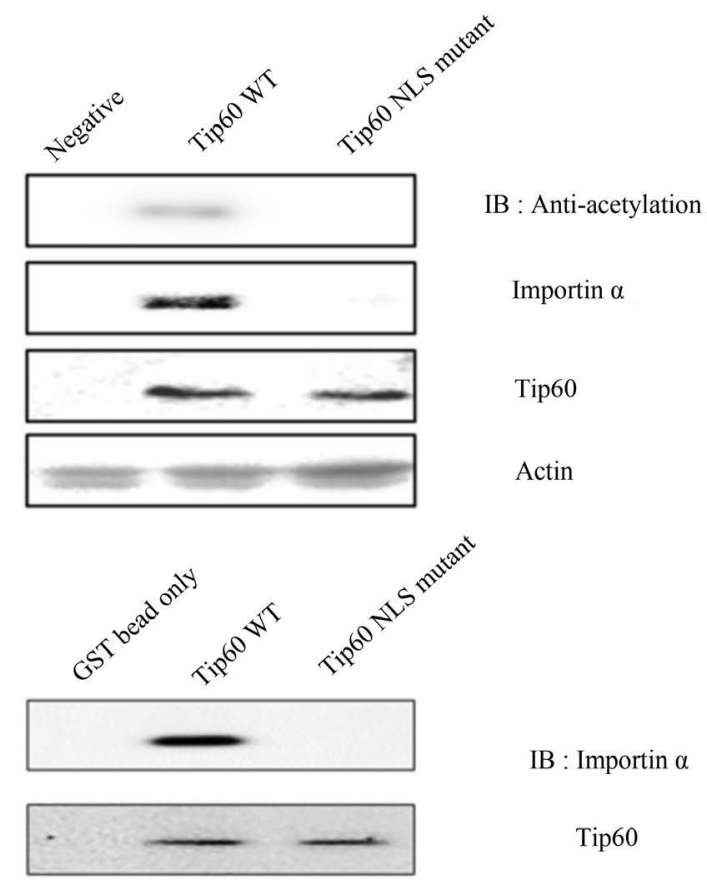


(C)

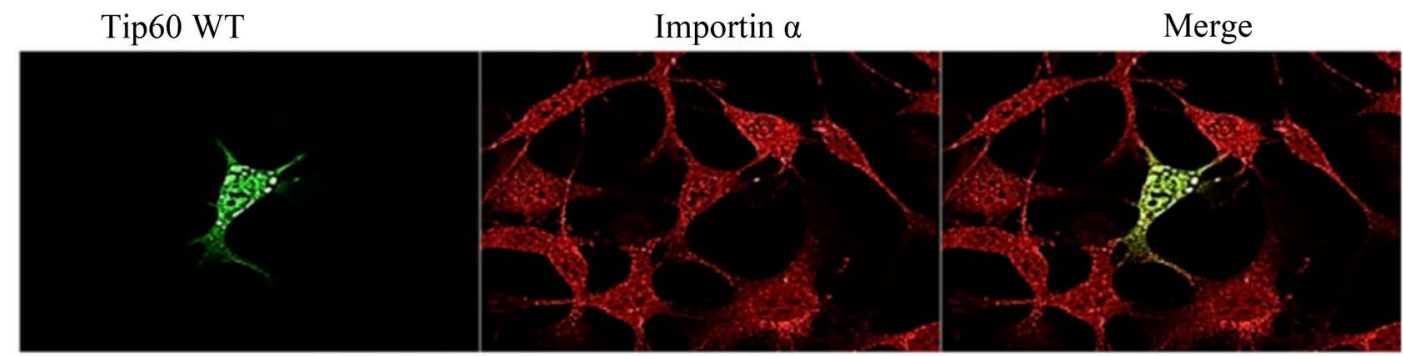

PCC between Ti60 and importina: $0.86+/-0.07(n=5)$

(D)

Tip60 NLS mutant

Importin $\alpha$

Merge

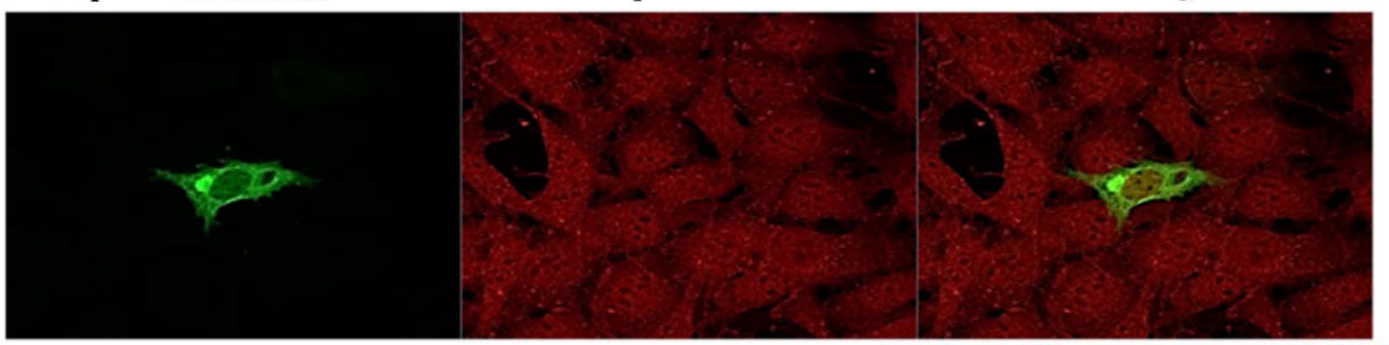

PCC between Ti60 and importina: $0.23+/-0.06(n=5)$

Figure 2. The subcellular localization of exogenous NLS mutant. (A) HEK293 cells were transiently transfected with expression vectors, EGFP-Tip60 WT, NLS mutant $\left({ }^{219} \mathrm{RKRK}^{222} \rightarrow{ }^{219} \mathrm{AAAA}^{222}\right)$. Following immunoprecipitation (IP) using an anti-EGFP antibody, acetylation (1st lane), importin $\alpha$ (2nd lane) or Tip60 (3rd lane) was detected with the immunoblot (IB) using an antibody against acetylation, importin $\alpha$ or Tip60. To monitor the cell lysate amount, anti-actin antibody was used for its western blot (4th lane). (B) In vitro pull down assay with the fusion protein of Tip60 (WT or NLS mutant). Whole cell lysates of HEK293 cells was incubated with $1 \mu \mathrm{g}$ of each glutathione agarose tagged recombinant Tip60 (WT or NLS mutant). The immunoblot was performed to detect importin $\alpha$ with its antibody (upper lane). The recombinant Tip60 (WT or NLS mutant) protein amount was monitored with the coomasaie blue staining (bottom lane). Confocal fluorescence micrographs of EGFP-Tip60, or NLS mutant $\left({ }^{219} \mathrm{RKRK}^{222} \rightarrow{ }^{219} \mathrm{AAAA}^{222}\right)$ in HEK293 cells. Importin $\alpha$ was visualized by immunofluorescence in fixed and permeablized cells using a polyclonal antibody against human an importin $\alpha$ and Alexa Fluor 568 conjugated donkey anti-rabbit IgG. The yellow pattern resulting from the merging of red and green colors indicates co-localization of both proteins at a specific region of the plasma membrane or cytoplasm, similar to the results obtained for endogenous Tip60 shown in Figure 1(C). All constructs were shown as green color and performed to determine whether it merged with Tip60. The transfected EGFP-Tip60 WT (detected in both the cytoplasm and the nucleus) was merged (yellow) with Tip60 nuclear (C). The transfected EGFP-NLS mutant $\left({ }^{219} \mathrm{RKRK}^{222} \rightarrow{ }^{219} \mathrm{AAAA}^{222}\right)$ was not found in the nuclear region, and was not merged with importin $\alpha$ in the nuclear (D).

To further confirm that the putative NLS motif $\left({ }^{210}\right.$ RRAVAAQPGRKRK $\left.{ }^{222}\right)$ of Tip60 could function as a classic NLS, importin $\alpha$ pull down assay was performed using GST-tagged Tip60 WT or NLS mutant fusion proteins expressed in E. coli. Our results revealed that both wild-type Tip60 fusion proteins pulled down importin $\alpha$ from HEK293 cell lysates in high quantities whereas its NLS mutant fusion proteins failed to pull down importin $\alpha$ (Figure 1(B)). This result was consistent with the co-immunoprecipitation result (Figure 1(A)). To better understand the function of the interaction between Tip60 and importin $\alpha$, confocal microscopic analysis was performed (Figure 2(C) and Figure 2(D)). 
Consistent with co-localization between endogenous Tip60 and importin $\alpha$ as shown in Figure 1(C), exogenous EGFP Tip60 WT (green) and importin $\alpha$ (red) were also co-localized together (yellow) in the nucleus (Figure 2(C)). However, exogenous EGFP-Tip 60 NLS mutant $\left({ }^{219} \mathrm{RKRK}^{222} \rightarrow{ }^{219} \mathrm{AAAA}^{222}\right.$; green $)$ did not merge or co-localize with importin $\alpha$ (red) in the nucleus (Figure 2(D)) probably due to mutations at the importin $\alpha$ binding site. Tip60 NLS mutant excluding the putative NLS sequence did not co-localized strongly with importin $\alpha$ in the nucleus either (Figure 2(D)). Furthermore, Tip60 mutant was less (1/4) self-acetylated than Tip60 WT (Figure 2(A)), suggesting that subcellular localization of Tip60 was influenced by its acetylation (Figure 2(D)). PCC between Tip60 WT and importin $\alpha$ was $0.86+/-0.07(\mathrm{n}=5)$ in Figure 2(C). That of Tip60 NLS mutant and importin $\alpha$ was reduced to $0.23+/-0.06(\mathrm{n}=5)$ in Figure 2(D). The decrease of PCC in Tip60 NLS mutant with importin $\alpha$ indicated that the co-existence (or interaction) of both proteins in cells was probably reduced to $1 / 4$ level of the value of Tip60 WT.

Comparing confocal results of Tip60 WT to those of NLS mutant, our data strongly demonstrated that importin $\alpha$ could interact with Tip60 through its putative consensus motif of Tip60. In addition, protein-protein interaction between Tip60 and importin $\alpha$ is required for the subcellular localization of Tip60 to the nucleus and its self-acetylation. These results (Figures 2(A)-(D)) demonstrated unequivocally that importin $\alpha$ could bind to Tip60 through the putative NLS motif of Tip60.

\subsection{Nuclear Localization of Cytoplasmic Protein Is Promoted by Fusion of Tip60 Motif}

As shown in Figure 1 and Figure 2, the putative NLS motif of Tip60 contributed to its physical interaction with importin $\alpha$ and its nuclear localization. To determine whether the putative NLS in Tip60 could interact with importin $\alpha$, Tip60 NLS $\left({ }^{210}\right.$ RRAVAAQPGRKRK ${ }^{222}$ ) motif was fused to YAP (Yes associated protein 65) 1-210 fragment C-terminal tail mainly localized in the cytoplasm based on our unpublished data [29]. After transfecting EGFP vector, EGFP YAP 1-210 fragment WT, or EGFP YAP 1-210 Tip60 NLS motif in HEK293 cells, each EGFP-fusion protein was purified with EGFP antibody. To monitor the expression level of Tip60 and its NLS mutant, western blot was also performed using anti-Tip60 Ab (Figure 3(C)). Yap 1-210 fragment fused with Tip60 NLS motif interacted with importin $\alpha$, although EGFP-Yap 1-210 fragment itself did not interact with importin $\alpha$. In addition, EGFP-Yap 1-210 was not localized in the nucleus. Our observation suggests that Tip60 motif is both necessary and sufficient as a NLS to facilitate the interaction between Tip60 and importin $\alpha$ (Figure 4).

\subsection{Effect of Cytoplasmic Localization of Tip60 on Cell Viability and its HAT Activity}

As shown in Table 1, NLS mutants significantly increased cell survival rate compared to EGFP-Tip60 WT or NLS mutant which interacted with importin $\alpha$. As 


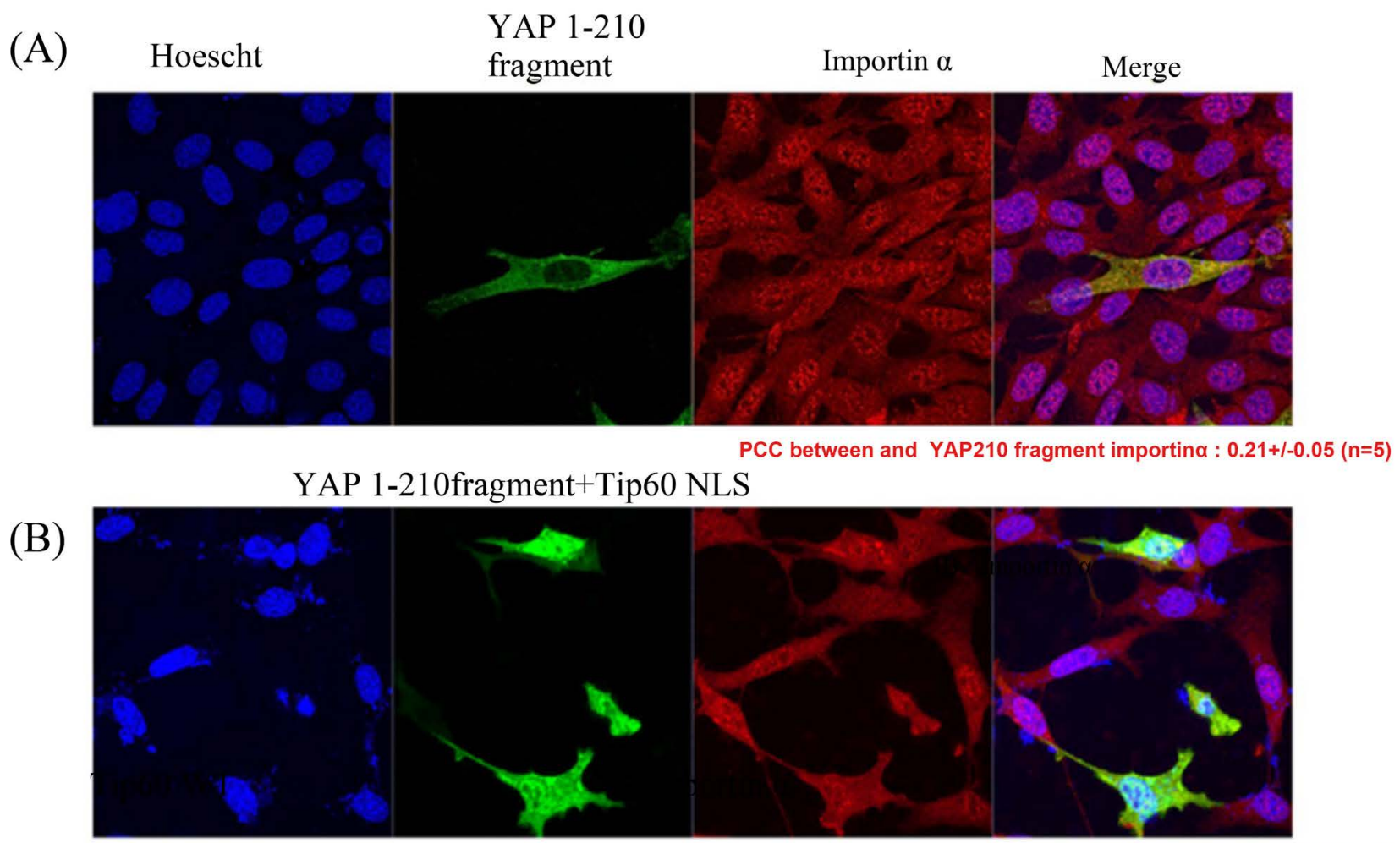

PCC between and YAP210 fragment importina : $0.86+1-0.07(n=5)$

(C)

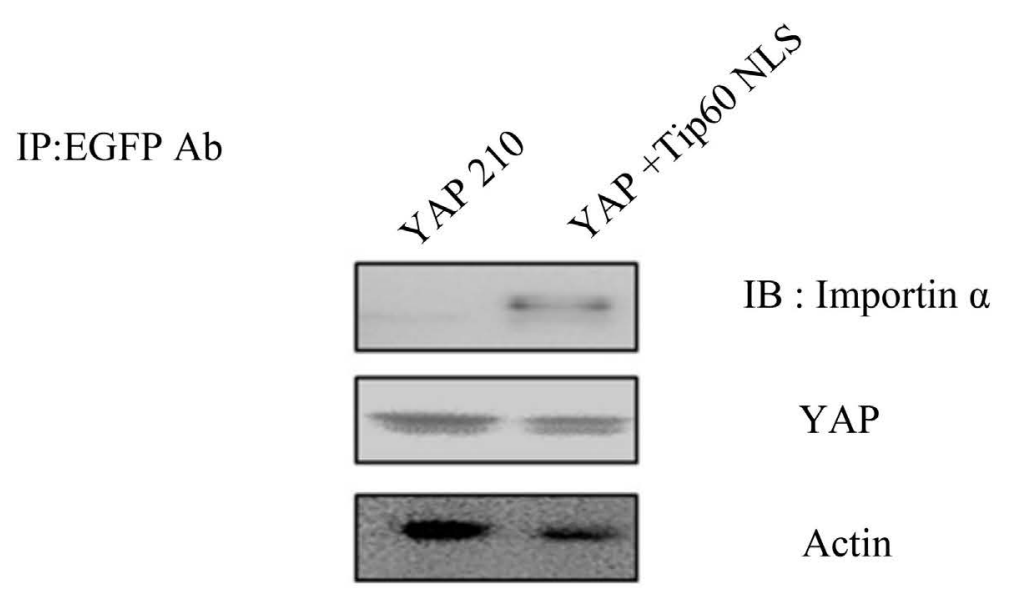

Figure 3. Nuclear localization of cytoplasmic protein is promoted by attachment of Tip60 ${ }^{210}$ RRAVAAQPGRKRK ${ }^{222}$ NLS motif. EGFP- YAP 1-210 fragment (only localized in the nuclear) or YAP 1-210 fragment fused with ${ }^{210}$ RRAVAAQPGRKRK ${ }^{222}$ NLS motif was transfected and confocal microscopic observation was conducted as described in the Material and Methods section. Importin $\alpha$ was visualized by immunofluorescence in fixed and permeablized cells using a polyclonal antibody against human importin $\alpha$ and Alexa Fluor 568 conjugated donkey anti-rabbit IgG (red color). The yellow pattern resulting from the merging of red and green colors indicates co-localization of both proteins at a specific region. The transfected EGFP-YAP 210 which is only detected in the cytoplasm was not merged with importin $\alpha$ in the nuclear (A). However, the transfected EGFP-YAP 1-210 fused with of Tip60 ${ }^{210}$ RRAVAAQPGRKRK ${ }^{222}$ NLS motif was found in the nuclear (B). To control the specificity of NLS mutant subcellular localization, unrelated NLS mutant control was also visualized (C). HEK293 cells were transiently transfected with expression vectors, EGFP-Tip60 WT, or NLS mutant $\left({ }^{219} \mathrm{RKRK}^{222} \rightarrow{ }^{219} \mathrm{AAAA}^{222}\right)$. Following immunoprecipitation (IP) using an anti-HA antibody, either importin $\alpha$ (top lane) or Tip60 (bottom lane) was detected by immunoblotting (IB) using an antibody against importin $\alpha$ or Tip60. YAP 210 fragment contains Tip60 $\left({ }^{210}\right.$ RRAVAAQPGRKRK $\left.{ }^{222}\right)$ motif in its C-terminal (YAP 210+Tip60 NLS; right lane). This fusion protein was strongly acetylated and pulled down with importin $\alpha$. 


\section{Cytoplasm Nuclear pore Nuclear}
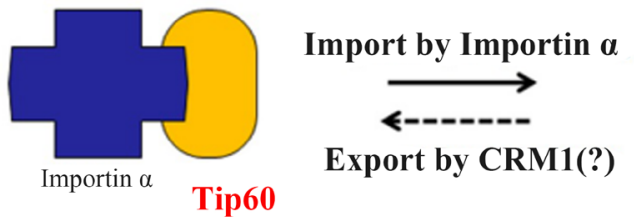

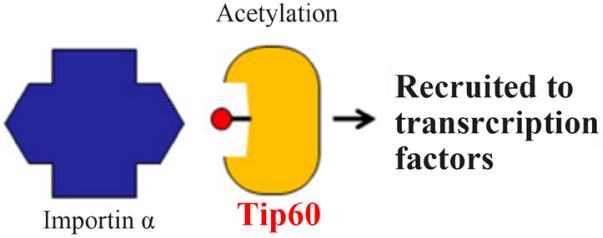

Self acetylation inhibits CRM1 binding and its export

Deaceylation by SIRT1.

Prolonged Nuclear retention by self acetylation

Figure 4. Import/export of Tip60 through its putative NLS and NES. Tip60 is imported/exported by importin $\alpha /$ CRM1 through its NLS or NES. The imported Tip60 seems to be auto acetylated, while the cytoplasmic Tip60 seems to be methylated. The auto acetylated Tip60 is profoundly localized into the nuclear, where it is recruited by to the transcriptional factors and operates its histone acetyltransferase activity to unwind the chromatin. Even the deacetylaon of Tip60 by SIRT1 is not clear yet, we assume that CRM1 exports Tip60 which is de (under)-acteylated by SIRT1 (or other enzyme). The acetylation of Tip60 seems to extend its duration time in the nuclear.

Table 1. The comparison of cell survival ratio of Tip60 WT with its NLS mutant. EGFP-Tip60 WT, its NLS mutant $\left({ }^{219} \mathrm{RKRK}^{222} \rightarrow{ }^{219} \mathrm{AAAA}^{222}\right)$, or EGFP vector was transfected and the rate of apoptosis measured by FACS. EGFP-NLS mutant, which was not dominantly localized into the nuclear was less effective on the cell apoptosis, compared to Tip60 WT constructs. The apoptotic effect of NLS mutant was reduced to 55\% of Tip60 WT.

\begin{tabular}{cc}
\hline Tip60 & Rate of apoptosis (\%) by FACS \\
\hline${ }^{\star} \mathrm{WT}$ & $12.16+/-3$ \\
${ }^{\star} \mathrm{NLS}$ mutant $\left({ }^{219} \mathrm{RKRK}^{222} \rightarrow{ }^{219} \mathrm{AAAA}^{222}\right)$ & $6.67+/-4$ \\
$($ Vector only) & $14.69+/-2$ \\
\hline
\end{tabular}

${ }^{*} \mathrm{P}<0.05$. Mean value of 5 repeats.

shown in Table 1, NLS mutant appeared to be 70\% less effective on HEK293 cell apoptosis than EGFP-Tip60 WT. Thus, nuclear localization of Tip60 seems to be required for its effect on cell viability. However, because NLS mutant $\left({ }^{219} \mathrm{RKRK}^{222} \rightarrow\right.$ ${ }^{219} \mathrm{AAAA}^{222}$ ) was less self-acetylated (Figure 2(A) upper lane), the effect of Tip60 on cell viability might be related to its HAT activity (Table 2). This suggests that proper localization of Tip60 is required for its effect on both cell viability and HAT activity. Tip60 NLS mutant which ablated its association with importin $\alpha$ showed both less apoptotic effect (Table 1 ) and less HAT activity (Table 2) than Tip60 WT. Although mutation $\left({ }^{219} \mathrm{RKRK}^{222} \rightarrow{ }^{219} \mathrm{AAAA}^{222}\right)$ of Tip60 might have affected both cell viability and its HAT activity, our results strongly suggest that its nuclear localization is a prerequisite for its tumor promoting function.

In conclusion, our results indicate that Tip60 can bind to importin $\alpha$ through its binding domain containing the putative NLS motif $\left({ }^{210}\right.$ RRAVAAQPGRKRK $\left.{ }^{222}\right)$ and that this interaction can regulate its function (HAT activity and apoptotic ability) by controlling its nuclear localization. 
Table 2. The comparison of HAT activity of Tip60 WT with its NLS mutant with ELISA. EGFP-Tip60 WT, its NLS mutant $\left({ }^{219} \mathrm{RKRK}^{222} \rightarrow{ }^{219} \mathrm{AAAA}^{222}\right)$, or EGFP vector was transfected and each HAT was measured by ELISA. The HAT of NLS mutant was reduced to $75 \%$ that of Tip60 WT.

\begin{tabular}{|c|c|}
\hline Tip60 & ELIA assay (relative activity; \%) \\
\hline${ }^{* *} \mathrm{WT}$ & $100+/-6$ \\
\hline${ }^{* *}$ NLS mutant $\left({ }^{219} \mathrm{RKRK}^{222} \rightarrow{ }^{219} \mathrm{AAAA}^{222}\right)$ & $74+/-5$ \\
\hline (Vector only) & $10+/-4$ \\
\hline
\end{tabular}

${ }^{* *} P<0.05$. Mean value of 10 repeats.

\section{Discussion}

The function of Tip60 in signal transduction has been most clearly characterized in the context of HAT signaling, in which it functions as a hub protein HAT [4] [13]. Results of the present study demonstrated that importin $\alpha$ could function as one Tip60 nuclear transporter. In addition, the putative Tip60 NLS is localized between chromodomain and $\mathrm{Zn}^{2+}$ finger based on site-directed mutagenesis analysis (Figure $1(\mathrm{~A})$ ).

Although our observation demonstrated that the interaction between Tip60 and importin $\alpha$ through NLS binding not only enhanced Tip60's HAT activity, but also controlled its subcellular localization, our findings also raised several questions regarding the interaction between Tip60 and importin $\alpha$. It remains unknown whether self-acetylation or Tip60's HAT activity was actually necessary for its functional interaction with importin $\alpha$ in vivo. Further experiments are clearly warranted in order to gain a greater understanding of the biological implication regarding the high degree of conservation of a well-defined importin $\alpha$ binding motif in Tip60. Moreover, the fashion and mechanisms that control the interaction betweenTip60 and importin $\alpha$ need to be evaluated under physiological conditions. It is currently unclear which kind of cellular signal can enhance or reduce the interaction between these two proteins. In addition, it remains to be determined whether Tip60 NLS mutation itself affects HAT and self-acetylation activity of Tip60 regardless of protein-protein interaction with importin $\alpha$. It is also necessary to ascertain whether post translational modification of importin $\alpha$ is required for the activation and/or regulation of Tip60 or for the interaction between Tip60 and importin $\alpha$.

At the present time, whether NLS mutation of Tip60 directly affects self-acetylation (Figure 2(A)) which is required for its HAT activity (Table 2) remains unclear. Tip60 NLS mutant which could not be self-acetylated was mainly found in the cytoplasm based on confocal analysis (Figure 2(A), Figure 2(D)). The blockade of nuclear localization of NLS mutant might be due to the inhibition of importin $\alpha$ binding on its middle domain by point mutation, not by self-acetylation (on Lys109, Lys113, Lys222, or Lys360) or its HAT activity [30]. Because Lys222 of Tip60 is overlapped with the putative NLS, self-acetylation may affect binding between importin $\alpha$ and Tip60 (Figure 1(A)). Even though it remains to be characterized whether self-acetylation is dependent on its import or 
export, nuclear localization of Tip60 seems to be dependent on acetylation at its lysine residues [30].

Results of this study demonstrated that importin $\alpha$ could bind to Tip60 through the putative NLS motif located between the chromodomain and $\mathrm{Zn}^{2+}$ finger domain of Tip 60 (Figure 1). This motif is found in all Tip60 family proteins available in GenBank (data not shown). Results on both self-acetylation activity and HAT activity of Tip60 revealed that these activities were inhibited upon engagement with importin $\alpha$ (Figure 3). Therefore, importin $\alpha$ might act as an agonistic to signal transduction involving Tip60. Although data of this study suggest that the importin $\alpha$ may function as a positive regulator of Tip60 signaling, the precise mechanism underlying the nuclear localization of Tip60 requires further characterization in order to gain better insight into the overall function of importin $\alpha$ signal transduction pathway (Figure 4). Tip60 also contains a putative PY-NLS $\left({ }^{271}\right.$ grhrlkpwyspypqeltt $\left.{ }^{290}\right)$ in front of its $\mathrm{Zn}^{2+}$ finger (Figure 1(A)) [1] [16] [28]. We cannot rule out the possibility that karyopherin $\beta$ might also contribute to the nuclear localization of Tip60 [1] [16] [29]. Further experiments are needed to demonstrate whether karyopherin $\beta$ also imports Tip60. If this is the case, what is its distinguishable role in Tip60 nuclear localization? However, PY-NLS recognized by karyopherin $\beta_{2}$ (Kap104p in yeast) is a larger linear signal that is quite diverse in sequence, in contrast to the canonical small monopartite cNLS [1] [16] [28].

We also observed that Tip60 was associated with chromosome region maintenance 1 (CRM1) for its export from the nucleus (unpublished data). The potential nuclear export sequence (NES) motif ( ${ }^{281}$ grhrlkpwyf spypqelttlpvlylcefcl $\left.{ }^{300}\right)$ in Tip60 is also noticed. This motif is similar to the consensus NES motif $\left(\Phi \mathrm{x}_{2-3} \Phi \mathrm{x}_{2-3} \mathrm{LxL} / \mathrm{I}\right)$. Tip60 export seems to be mediated by CRM1 [1] [30]. Treatment with Leptomycin B, an inhibitor of CRM1, enhanced Tip60 accumulation in the nucleus (our unpublished data). Therefore, CRM1 might mediate Tip60 export through its NES motif [1] [30]. However, detail mechanisms involved in Tip60 import/ export remain to be characterized.

Interestingly, self-acetylation on lysine residue 301 which is overlapped with CRM1 binding seems to inhibit its export by CRM1 (Figure 4 and Figure 2(A) upper lane) [1] [28] [30]. The nuclear retained Tip60 might be recruited to transcriptional factors (or other proteins) for unfolding chromatin. Yes-associated protein (YAP, a Hippo signal pathway intermediator, co-transcriptional factor) also binds to Tip60 through its WW domain in the nucleus (unpublished data). However, the detailed mechanism of how Tip60 is (de)acetylated and biological significance underlying this modification remain to be characterized. In the future, characterization of Tip60 NLS may contribute to new strategies to understand and manipulate its cellular actions in gene expression and apoptosis as well as its involvement in human diseases [4] [13] [30] [31].

\section{Acknowledgements}

This work was supported by a National Research Foundation of Korea grant 
(NRF-2013R1A1A4A01005522 and NRF-2016R1D1A3B03934269) to S. S. Kang. Shin is a Korean Research fellowship recipient (NRF-2014R1A1A2009622).

\section{Conflicts of Interest}

The authors declare no conflicts of interest regarding the publication of this paper.

\section{References}

[1] Kamine, J., Elangovan, B., Subramanian, T., Coleman, D. and Chinnadurai, G. (1996) Identification of a Cellular Protein That Specifically Interacts with the Essential Cysteine Region of the HIV-1 Tat Transactivator. Virology, 216, 357-366.

[2] Yamamoto, T. and Horikoshi, M. (1997) Novel Substrate Specificity of the Histone Acetyltransferase Activity of HIV-1-Tat Interactive Protein Tip60. The Journal of Biological Chemistry, 272, 30595-30598. https://doi.org/10.1074/jbc.272.49.30595

[3] Lemercier, C., Legube, G., Caron, C., Louwagie, M., Garin, J., Trouche, D. and Khochbin, S. (2003) Tip60 Acetyltransferase Activity Is Controlled by Phosphorylation. The Journal of Biological Chemistry, 278, 4713-4718. https://doi.org/10.1074/jbc.M211811200

[4] Sapountzi, V., Logan, I.R. and Robson, C.N. (2006) Cellular Functions of TIP60. The International Journal of Biochemistry and Cell Biology, 38, 1496-1509. https://doi.org/10.1016/j.biocel.2006.03.003

[5] Col, E., Caron, C., Chable-Bessia, C., Legube, G., Gazzeri, S., Komatsu, Y., Yoshida, M., Benkirane, M., Trouche, D. and Khochbin, S. (2005) HIV-1 Tat Targets Tip60 to Impair the Apoptotic Cell Response to Genotoxic Stresses. The EMBO Journal, 24, 2634-2645. https://doi.org/10.1038/sj.emboj.7600734

[6] Bock, F.J., Tanzer, M.C., Haschka, M.D., Krumschnabel, G., Sohm, B., Goetsch, K., Kofler, R. and Villunger, A. (2015) The p53 Binding Protein PDCD5 Is Not Rate-Limiting in DNA Damage Induced Cell Death. Scientific Reports, 5, Article No. 11268. https://doi.org/10.1038/srep11268

[7] Cui, H., Li, X., Han, C., Wang, Q.E., Wang, H., Ding, H.F., Zhang, J. and Yan, C. (2016) The Stress-Responsive Gene ATF3 Mediates Dichotomous UV Responses by Regulating the Tip60 and p53 Proteins. The Journal of Biological Chemistry, 291, 10847-10857. https://doi.org/10.1074/jbc.M115.713099

[8] Lee, E.J., Shin, S.H., Hyun, S., Chun, J. and Kang, S.S. (2012) Endoplasmic Reticulum (ER) Stress Enhances Tip60 (A Histone Acetyltransferase) Binding to the Concanavalin A. The Open Biochemistry Journal, 6, 1-10. https://doi.org/10.2174/1874091X01206010001

[9] Shin, S.H. and Kang, S.S. (2013) Phosphorylation of Tip60 Tyrosine 327 by Abl Kinase Inhibits HAT Activity through Association with FE65. The Open Biochemistry Journal, 7, 66-72. https://doi.org/10.2174/1874091X20130622002

[10] Hilfiker, A., Hilfiker-Kleiner, D., Pannuti, A. and Lucchesi, JC. (1997) Mof, A Putative Acetyl Transferase Gene Related to the Tip60 and MOZ Human Genes and to the SAS Genes of Yeast, Is Required for Dosage Compensation in Drosophila. The EMBO Journal, 16, 2054-2060. https://doi.org/10.1093/emboj/16.8.2054

[11] Kimura, A. and Horikoshi, M. (1998) Tip60 Acetylates Six Lysines of a Specific Class in Core Histones in Vitro. Genes to Cells. Devoted to Molecular and Cellular Mechanisms, 3, 789-800. https://doi.org/10.1046/j.1365-2443.1998.00229.x 
[12] Legube, G., Linares, L.K., Lemercier, C., Scheffner, M., Khochbin, S. and Trouche, D. (2002) Tip60 Is Targeted to Proteasome-Mediated Degradation by Mdm2 and Accumulates after UV Irradiation. The EMBO Journal, 21, 1704-1712. https://doi.org/10.1093/emboj/21.7.1704

[13] Fu, M., Wang, C., Zhang, X. and Pestell, R.G. (2004) Acetylation of Nuclear Receptors in Cellular Growth and Apoptosis. Biochemical Pharmacology, 68, 1199-1208. https://doi.org/10.1016/j.bcp.2004.05.037

[14] Logan, I.R., Sapountzi, V., Gaughan, L., Neal, D.E. and Robson, C.N. (2004) Control of Human PIRH2 Protein Stability: Involvement of TIP60 and the Proteosome. The Journal of Biological Chemistry, 279, 11696-11704. https://doi.org/10.1074/jbc.M312712200

[15] Zhuge, C., Chang, Y., Li, Y., Chen, Y. and Lei, J. (2011) PDCD5-Regulated Cell Fate Decision after Ultraviolet-Irradiation-Induced DNA Damage. Biophysical Journal, 101, 2582-2591. https://doi.org/10.1016/j.bpj.2011.10.044

[16] Chook, Y.M. and Blobel, G. (2001) Karyopherins and Nuclear Import. Current Opinion in Structural Biology, 11, 703-715. https://doi.org/10.1016/S0959-440X(01)00264-0

[17] Okada, N., Ishigami, Y., Suzuki, T., Kaneko, A., Yasui, K., Fukutomi, R. and Isemura, M. (2008) Importins and Exportins in Cellular Differentiation. Journal of Cellular and Molecular Medicine, 12, 1863-1871. https://doi.org/10.1111/j.1582-4934.2008.00437.x

[18] Christie, M., Chang, C.W., Rona, G., Smith, K.M., Stewart, A.G., Takeda, A.A., Fontes, M.R., Stewart, M., Vertessy, B.G., Forwood, J.K. and Kobe, B. (2016) Structural Biology and Regulation of Protein Import into the Nucleus. Journal of Molecular Biology, 428, 2060-2090. https://doi.org/10.1016/j.jmb.2015.10.023

[19] Soniat, M. and Chook, Y.M. (2015) Nuclear Localization Signals for Four Distinct Karyopherin- $\beta$ Nuclear Import Systems. The Biochemical Journal, 468, 353-362. https://doi.org/10.1042/BJ20150368

[20] Gilchrist, D. and Rexach, M. (2003) Molecular Basis for the Rapid Dissociation of Nuclear Localization Signals from Karyopherin $\alpha$ in the Nucleoplasm. The Journal of Biological Chemistry, 278, 51937-51949. https://doi.org/10.1074/jbc.M307371200

[21] Hariton-Gazal, E., Friedler, D., Friedler, A., Zakai, N., Gilon, C. and Loyter, A. (2002) Inhibition of Nuclear Import by Backbone Cyclic Peptidomimetics Derived from the HIV-1 MA NLS Sequence. Biochimica et Biophysica Acta, 1594, 234-242. https://doi.org/10.1016/S0167-4838(01)00306-5

[22] Gorlich, D., Pante, N., Kutay, U., Aebi, U. and Bischoff, F.R. (1996) Identification of Different Roles for RanGDP and RanGTP in Nuclear Protein Import. The EMBO Journal, 15, 5584-5594. https://doi.org/10.1002/j.1460-2075.1996.tb00943.x

[23] Percipalle, P., Clarkson, W.D., Kent, H.M., Rhodes, D. and Stewart, M. (1997) Molecular Interactions between the Importin $\alpha / \beta$ Heterodimer and Proteins Involved in Vertebrate Nuclear Protein Import. Journal of Molecular Biology, 266, 722-732. https://doi.org/10.1006/jmbi.1996.0801

[24] Floer, M., Blobel, G. and Rexach, M. (1997) Disassembly of RanGTP-Karyopherin $\beta$ Complex, an Intermediate in Nuclear Protein Import. The Journal of Biological Chemistry, 272, 19538-19546. https://doi.org/10.1074/jbc.272.31.19538

[25] Pries, R., Bomeke, K., Draht, O., Kunzler, M. and Braus, G.H. (2004) Nuclear Import of Yeast Gcn4p Requires Karyopherins Srp1p and Kap95p. Molecular Genetics and Genomics: MGG, 271, 257-266. https://doi.org/10.1007/s00438-003-0955-7

[26] Fries, T., Betz, C., Sohn, K., Caesar, S., Schlenstedt, G. and Bailer, S.M. (2007) A 
Novel Conserved Nuclear Localization Signal Is Recognized by a Group of Yeast Importins. The Journal of Biological Chemistry, 282, 19292-19301.

https://doi.org/10.1074/jbc.M700217200

[27] Laemimi, U.K. (1970) Cleavage of Structural Proteins during the Assembly of the Head of Bacteriophage T4. Nature, 227, 680-685. https://doi.org/10.1038/227680a0

[28] Sudol, M. (1994) Yes-Associated Protein (YAP65) Is a Proline-Rich Phosphoprotein That Binds to the SH3 Domain of the Yes Proto-Oncogene Product. Oncogene, 9, 2145-2152.

[29] Yang, C., Wu, J. and Zheng, Y.G. (2012) Function of the Active Site Lysine Autoacetylation in Tip60 Catalysis. PLoS ONE, 7, e32886. https://doi.org/10.1371/journal.pone.0032886

[30] Shin, S.H., Lee, E.J., Chun, J., Hyun, S. and Kang, S.S. (2015) ULK2 Ser 1027 Phosphorylation by PKA Regulates Its Nuclear Localization Occurring through Karyopherin Beta 2 Recognition of a PY-NLS Motif. PLOS ONE, 10, e0127784. https://doi.org/10.1371/journal.pone.0127784

[31] Nayak, G. and Cooper, G.M. (2012) p53 Is a Major Component of the Transcriptional and Apoptotic Program Regulated by PI 3-Kinase/Akt/GSK3 Signaling. Cell Death and Disease, 3, e400. https://doi.org/10.1038/cddis.2012.138

\section{Abbreviations}

HAT: histone acetyltransferases

cNLSs: nuclear classic localization signals

FBS: fetal bovine serum

CRM: chromosome region maintenance 1

NES: nuclear export sequence

IP: immunoprecipitation

IB: using an anti-importin $\alpha$ antibody, an immunoblot 